\title{
Reference. $\begin{aligned} & \text { NBS } \\ & \text { Publi- } \\ & \text { cations }\end{aligned}$ \\ NBSIR 83-2646
}

\section{Characterization of Microwave Window Materials}

\author{
U.S. DEPARTMENT OF COMMERCE \\ National Bureau oi Standards \\ National Measurement Laboratory \\ Center for Materials Science \\ Inorganic Materials Division \\ Washington, DC 20234
}

May-October :982

Interim Report

Issued January 1983

Task. Code: NggCAXAH, Missile Systems; X-P.ay Hardiness

Work Unit: 00043, Mater:als Characterization

Drepared for

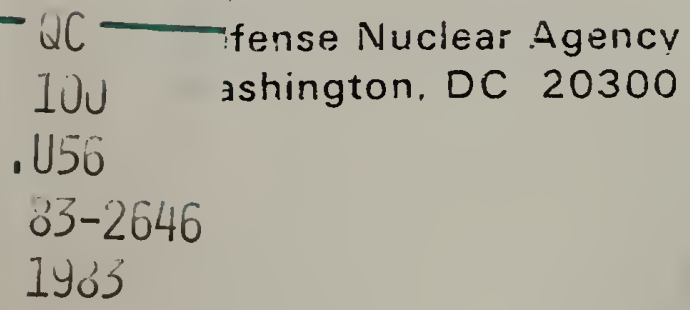





\section{CHARACTERIZATION OF MICROWAVE WINDOW MATERIALS}

H.P.R. Frederikse, A.L. Dragoo, and A.H. Kahn

U.S. DEPARTMENT OF COMMERCE

National Bureau of Standards

National Measurement Laboratory

Center for Materials Science

Inorganic Materials Division

Washington, DC 20234

W.R. Hosler

WRH Co.

Poolesville, MD

May-October 1982

Interim Report

Task Code: NggQAXAH/Missile Systems/X-Ray Hardness

Work Unit: 00043/Materials Characterization

Prepared for

Defense Nuclear Agency

Washington, DC 20300

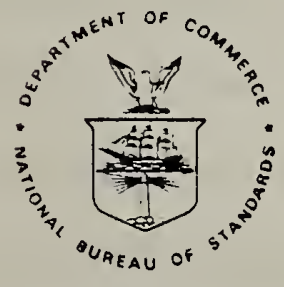

U.S. DEPARTMENT OF COMMERCE, Malcolm Baldrige, Secretary NATIONAL BUREAU OF STANDARDS, Ernest Ambler, Director 

Characterization of Microwave Window Materials

Interim Report

May-October 1982

\section{Introduction}

Since February 1982, NBS has been engaged in a project concerned with the properties of Boron Nitride. Hexagonal BN is a promising candidate for use as a microwave window material. The window will reach temperatures in the neighborhood of $3000 \mathrm{~K}$ for short periods of time. It is therefore important to know the physical and chemical properties of $\mathrm{BN}$ in this range. The focus of this project is on the electrical d.c. conductivity of $B N$ and on its dielectric behavior when subjected to microwave radiation at very high temperatures.

The first four months (Jan.-April) have been used to explore various experimental approaches to the measurement of the electrical resistance and the temperature in the presence of large electrical and thermal leaks. At the same time, computer programs were developed to calculate the dielectric parameters of $\mathrm{BN}$ in the low-GHz frequency range.

\section{Electrical Conductivity}

\section{Sample Configuration}

Although hexagonal boron nitride possibly is the best electrical insulator known up to $2000{ }^{\circ} \mathrm{C}$, above this temperature the conductivity rapidly increases to values in excess of $10^{-3} \mathrm{ohm}^{-1} \mathrm{~cm}^{-1}$. This conduction probably is intrinsic in rature. In order to prevent degradation and nonstoichiometry due to loss of nitrogen, which cou?d cause large extrinsic conduction, it is necessary to perform high temperature 
measurements in nitrogen gas. According to the literature [1], the equilibrium vapor pressure of $\mathrm{N}_{2}$ over $B N$ is $0.1 \mathrm{~atm}$ at $2265^{\circ} \mathrm{C}, 1.0 \mathrm{~atm}$ at $2505^{\circ} \mathrm{C}$, and $3.0 \mathrm{~atm}$ at $2626{ }^{\circ} \mathrm{C}$.

As stated in the first Progress Report (May 1982), above $2000{ }^{\circ} \mathrm{C}$ one observes a leakage conductance--probabiy due to gas conduction-which reaches values comparable to that of the BN sample. Consequently, it is necessary to devise an experimental arrangement which avoids the problem of the large leakage conduction path. One solution is a geometry in which the sample is isolated from the nitrogen gas (Scheme $\# 1$ ). Another arrangement is a guard ring configuration whereby the sample and sample leads are electrically shielded from the large leak-currents to ground (Scheme \#2).

Scheme \#1.

For this approach, the insulation of the sample from the gas is provided largely by boron nitride at a lower temperature, as shown in Fig. 1. The actial $B N$ sample is confined mainly to the volume between the tungsten electrodes. This "sample" is part of a BN cylinder which is positioned at the access opening of the lowest of the upper eight heat shields. Above this level, the temperature drops rapidly. Consequently, the boron nitride cylinder above the top electrode has a high resistance and contributes little to the conduction path. The bottom turigsten electrode is held in place by a threaded BN plug. Cue to this geometry, it is difficult to transiate the resistance, $R$, of the sample into resistivity. However, a rough estimate indicates that the numerical value of the resistivity is of the order of $1.5 \mathrm{R}$ and certainiy does not exceed $2.0 \mathrm{R}$. 
In the first run, the temperature of the sample was measured by a $W(3 \% \operatorname{Re})$ vs. W(25\% Re) thermocouple attached to the top electrode. The temperature in the center of the furnace was determined by means of a W( $5 \%$ Re) vs. W( $26 \% \operatorname{Re})$ thermocouple introduced through the bottom of the furnace and by means of a pyrometer sighted through a viewing port on the side of the furnace. The readings of the pyrometer and of the thermocouple in the center of the furnace agreed within $40-50{ }^{\circ} \mathrm{C}$ up to $2200{ }^{\circ} \mathrm{C}$; beyond this temperature, the latter was retracted from the furnace.

The sample used in the first run was cut from a small block of high purity boron nitride (HPBN-TS1251; Union Carbide). The temperature at the center of the furnace was raised to a maximum of $2480^{\circ} \mathrm{C}$, as indicated by the pyrometer. At this point, the sample temperature lagged behind considerably: $T=1850{ }^{\circ} \mathrm{C}$ was the nighest reading. A11 measurements were made with an $\mathrm{N}_{2}$ pressure of $1.0 \mathrm{~atm}$. Results of this first run are shown in Fig. 2.

In order to raise the sample temperature in the next run, the boron nitride cylinder was made somewhat longer, such that the top electrode was located $1.0 \mathrm{~cm}$ below the lowest horizontal heat shield. This made it possible to reach a higher sample temperature of $2021{ }^{\circ} \mathrm{C}$ when the maximum temperature in the center of the furnace was $2410{ }^{\circ} \mathrm{C}$, as indicated by the pyrometer. Conductivity measurements were made at three different pressures of the nitrogen gas: $1.0,2.0$, and $3.0 \mathrm{~atm}$. Results of this second run are also presented in Fig. 2.

Electrical measurements during the first run were terminated when the tungsten wire failed between the sample and the $B N$ insulator which 
extended through the viewing port into the hot zone of the furnace (see

Fig. 1). An attempt to push the furnace temperature above $2500{ }^{\circ} \mathrm{C}$ resulted in considerable effervescence and partial destruction of the end of the $B N$ insulator. The second run was terminated at a furnace temperature of about $2500{ }^{\circ} \mathrm{C}$ when the wires of the lower thermocouple on the sample broke.

Two materials failure modes are indicated by these results. The first mode is the decomposition of $B N$ at temperatures in excess of $2500{ }^{\circ} \mathrm{C}$ under a pressure of $1.0 \mathrm{~atm}$ of $\mathrm{N}_{2}$. This observation is in agreement with the literature value of $2505^{\circ} \mathrm{C}$ [1]. No decomposition of BN was evident, as expected, in the second run when the pressure was increased to $3.0 \mathrm{~atm}$ of $\mathrm{N}_{2}$.

The second mode appears to occur through a reaction between the tungsten wires and the BN parts. After each experiment, the BN sample and insulators were inspected radiographically. Radiographs of sample and insulators are shown in Fig. 3. The multiple breaks and balling up of the tungsten wire suggest that the wire failed by forming a liquid phase. This is in agreement with the $W-B$ phase diagram [2]: $W_{2} B_{5}$ melts at $2365{ }^{\circ} \mathrm{C}$ and excess boron lowers the melting temperature to $\sim 2000{ }^{\circ} \mathrm{C}$ (Fig. 4). When the amount of tungsten exceeds the BN present ( $W>50$ percent), the melting points of the $W-B$ compounds and solid solutions are $2600{ }^{\circ} \mathrm{C}$ or higher. Consequently, more massive pieces of tungsten (rods and discs), in contact with $B N$, should not be subject to disintegration if one keeps the temperature below $\sim 2600{ }^{\circ} \mathrm{C}$. Scheme \#2

In this arrangement (Fig. 5), the sample is located near the center of the furnace suspended from a tungsten tube, which acts as an elec- 
trical shield. A "lip" at the bottom of this shield functions as the guard ring. The sample is provided with two tungsten discs; these are the electrodes for measuring the resistance of the $\bar{B} N$ sample. A11 these parts are heid together by two $B N$ rings, threaded on the cylindrical sample.

The sample resistance, $R_{x}$, is measured by means of an OP-AMP circuit [3]; $R_{x}$ is deduced from the following expression:

$$
\frac{R_{x}}{E_{j}}=-\frac{R_{f}}{E_{0}},
$$

where: $\quad R_{x}=$ sample resistance,

$E_{j}=$ applied voltage,

$R_{f}=$ feedback resistance, and

$E_{0}=$ output voltage.

The OP-AMP circuit plays the role of a very sensitive nuliing device (OP-AMP A). The circuit is strictly d.c.; OP-AMP's $B$ and $C$ are filters to prevent any intrusion from low frequency a.c. sources.

The shield and the main furnace are at ground potential; point $P$, between $R_{x}$ and $R_{f}$, is only a few microvolts above ground. Consequentiy, no leakage can occur between the top lead to the sample and its surroundings. Possible leakage paths from the lower sample lead to the grounded furnace body are not part of the main circuit (as long as $\varepsilon_{i}$ does not vary) and, hence, are not being measured.

Conductivity measurements on several $\mathrm{BN}$ and BN-containing samples are in progress. 


\section{Theory of Microwave Window Transmission}

\section{General Considerations}

Under the conditions of atmospheric reentry, the ARV microwave window material is subjected to most severe environmental conditions. The very high outer surface temperature leads to ablation of the material and the flow of a heat pulse into the window. The temperatures reached are sufficiently high that the thermal generation of conducting carriers is sufficient to cause significant microwave absorption (and reflectance) in many candidate materials. The processes induced by reentry are so rapid that the temperature distribution and carrier concentrations are nonuniform.

Complete modeling of the transmissivity of a microwave window would require a knowledge of the ablation rate, the temperature profile, and the enhancement of electrical conductivity at all times during the reentry and also the effects of the external plasma of ionized gases. In this part of the project, we address the more limited question of finding the effects of the intrinsic conduction processes on the applicability of boron nitride for window use. We devote the principal effort to the calculation of the microwave transmission through window material at high temperatures, with a steep temperature gradient and with thermal excitation of conductivity.

The calculations we perform are based on the model illustrated in Fig. 6. Microwave radiation is incident from the left. Significant reflection (to be calculated) occurs; the radiation in the window undergoes multiple internal reflections and a transmitted wave emerges, propagating to the right. As the window ablates, the thickness of the 
window decreases, leading to interference oscillations in the transmissivity. An additional complication is that, for the conductivities and the microwave frequencies that are used, the electromagnetic skin depth and the wavelength in the window are smaller than the thickness of the hot layer at the outer surface. Thus, we must solve the probiem of electromagnetic radiation passing through a medium with parameters that are changing slowly with respect to the wavelength in the medium.

Because of the immediate availability of suitable computer programs, we felt that direct numerical calculation was the simplest way to treat this problem correctly. Approximations utilizing the WKBJ [4] method could have been used, but computer usage would probably have been necessary anyway.

\section{Method of Calculation}

The propagation of an electromagnetic wave in a medium having a spatially varying electrical conductivity is described by Maxwe17's equations. If we assume that the material variation is a function of one coordinate, $z$; i.e., that the medium is stratified and that the radiation propagate is parallel to the $z$-direction, then the wave is described by the pair of coupled linear differential equations:

$$
\begin{aligned}
\frac{d E}{d z} & =-i k Z_{0} H, \\
Z_{0} \frac{d H}{d z} & =-i k \varepsilon(z) E .
\end{aligned}
$$

In the above, $E$ and $H$ are the electric and magnetic field intensities, which are mutually perpendicular, and both are transverse to the $z$ - 
direction. Sinusoidal monochromatic fields of angular frequency, w, are assumed; the convention for the time variation is by use of the understood factor, $e^{-i \omega t} . z_{0}$ is the impedance of free space, and $k$ is the wave vector equal to the reciprocal wavelength times $2 \pi$. The complex dielectric constant, $\varepsilon$, is given by,

$$
\varepsilon(z)=\varepsilon_{0} \kappa_{e}+i \sigma(z) / \omega,
$$

where $\varepsilon_{0}$ is the permittivity of free space, $\kappa_{e}$ is the relative dielectric permittivity of the window material, and $\sigma(z)$ is the spatially varying electrical conductivity.

In the above, SI units are used exclusively. The numerical solutions of Eqs. (1.a) and (1.b), subject to specially imposed boundary conditions, enable one to compute the characteristic matrix of the material. By using standard methods [5], which we shall not reproduce here, the transmissivity and reflectivity of material of arbitrary dielectric profile can be obtained once the characteristic matrix is computed.

The problem is thus completely specified if we give the temperature profile and the dependence of conductivity on temperature. For the temperature profile, we use the values reported by the TRW Corporation [6] on data for hot pressed boron nitride. The profile is given approximately by the exponential form,

$$
T=T_{0} e^{-\alpha z},
$$


where $\alpha=0.216 \mathrm{~cm}^{-1}$ is the decay constant and $T_{0}$ is the surface temper ature. For the conductivity of $\mathrm{BN}$, we use the loss tangent data compiled by Hathaway et al. [7]. The loss tangent, $\tan \sigma$, is related to the conductivity by,

$$
\tan \sigma=\frac{\sigma}{\varepsilon_{0}^{k} e^{\omega}}
$$

Here, $\mathrm{K}_{\mathrm{e}}$ is the dielectric constant of BN, taken as 3.1. Their data [7] are fitted well by the Arrhenius form,

$$
\sigma(T)=\sigma_{0} e^{-\Delta / k T}
$$

where the prefactor, $\sigma_{0}=1.63 \times 10^{3} \Omega^{-1} \mathrm{~cm}^{-1}$, and $\Delta=3.08 \mathrm{eV}$, are consistent with a band gap of $6.0 \mathrm{eV}$. For a surface temperature of $3150 \mathrm{~K}$, the conductivity at the surface would be $2.0 \mathrm{~s}^{-1} \mathrm{~cm}^{-1}$; very close to the values extrapolated from the measurements of this report (see Fig. 2).

\section{Results of the Calculations and Discussion}

In Fig. 7 through Fig. 10, we show the calculated transmissivities of boron nitride windows as a function of thickness for a range of surface temperatures. The curves have common characteristics which we now shall describe. The transmissivity falls very rapidly as window thickness increases from zero. The curves all show that the principal effects of the conduction processes occur in the outer one or two millimeters of the hot face. All curves show a near-sinusoidal oscillation with thickness, which is characteristic of the multiple internal reflec- 
tions that occur in the cool part of the window. The percent transmissivity increases as the surface temperature is reduced, to as high as nine percent for $T_{0}=2850 \mathrm{~K}$.

In the various cases studied, the reflectivity varied from 80 percent to 85 percent, and the absorptivity varied from 10 percent to 15 percent. This demonstrates the mirror-like properties of the outer hot surface of the window.

The oscillatory dependence upon thickness suggests that one could observe, at $10 \mathrm{GHz}$, a series of transmissivity peaks as the ablation process proceeds. We consider the peak values as the maximum obtainable transmissions, for if the window were coupled to a continuously tuned cavity the interference cancellation could be removed.

The conclusion to be drawn from these calculations is that, at the considered reentry conditions, the transmissivity of intrinsic boron nitride windows at $10 \mathrm{GHz}$ will have an upper limit of 10 percent; i.e., the one-way loss will be $10 \mathrm{~dB}$ or greater. Some improvement could be obtained by altering the material composition and form in such a way as to reduce the surface temperature or to increase the temperature gradient. Calculations of this type should be extended to materials, such as woven $\mathrm{BN}-\mathrm{SiO}_{2}$ fiber composites and other candidate materials. Also, an improved theory of the temperature profile should be developed. 


\section{References}

1. Gmelin Handbuch der Anorg. Chemie, Band 13: Borverbindungen, Teil 1; Springer Verlag, Berlin-New York, 1974, p. 70.

2. "Compendium of Phase Diagram Data", E. Rudy, AFML-TR-65-2, Part V, Air Force Matls. Lab., WPAFB, Dayton, OH, 1969.

3. "Operational Amplifiers", J. G. Graeme, G. E. Tobey, and L. P. Huelsman, McGraw-Hi11 Book Co., New York, 1971.

4. "Light Reflection From Films of Cuntinuously Varying Refractive Index", J. Jacobsson, Progress in Optics, E. Wolf, Ed., North Holland, Amsterdam, 1966; Vol. V.

5. "Principles of Optics", M. Born and E. Wolf, Pergamon Press, Oxford, 1965; pp. 51-66.

6. "The Prediction and Measure of Dielectric Properties and RF Transmission Through Ablating BN Antenna Windows", K. Golden (TRW), B. Hanawalt (AVCO), and W. Ossmann (AVCO), AIAA-81-1085, 16th Thermophysics Conf., Palo Alto, CA, 1981.

7. "Microwave Loss at High Temperatures in Radome Materials", K. Hathaway, J. Cullen, and A. A. Campolattaro, Nav. Surf. Weapons Ctr., TR-80-555, Apri1 1981. 


\section{Figure Captions}

1. Boron Nitride sample with internal electrodes (Scheme 1):

$A, B$--tungsten electrodes

T--W(3Re) vs. W(25Re) thermocouple

H--heater

S--radiation shields

2. Resistance of $B N$ as a function of reciprocal temperature:

$$
\begin{aligned}
& \text { 0--Run I } \\
& x \text {--Run II; } P_{N_{2}}=1.0 \mathrm{~atm} \\
& \text { o--Run II; } P_{N_{2}}=3.0 \mathrm{~atm}
\end{aligned}
$$

3. Radiographs of $\mathrm{BN}$ sample.

4. Phase Diagram of $W-B$ system.

5. Schematic of sample holder, furnace, and OP-AMP circuit (Scheme 2).

6. Illustration of the model for calculating the microwave transmissivity of $B N$ windows.

7. Calculated transmissivity of $B N$ microwave windows as a function of thickness for $1.0 \mathrm{GHz}$ radiation and a surface temperature of $3150 \mathrm{~K}$ $\left(2877^{\circ} \mathrm{C}\right)$.

8. Calculated transmissivity of $B N$ microwave windows as a function of thickness for $f=10.0 \mathrm{GHz}$ and $T=3150 \mathrm{~K}\left(2877^{\circ} \mathrm{C}\right)$.

9. Calculated transmissivity of BN microwave windows as a function of thickness for $f=10.0 \mathrm{GHz}$ and $T=3000 \mathrm{~K}\left(2727^{\circ} \mathrm{C}\right)$.

10. Calculated transmissivity of $B N$ microwave windows as a function of thickness for $f=10.0 \mathrm{GHz}$ and $T=2850 \mathrm{~K}\left(2577^{\circ} \mathrm{C}\right)$. 


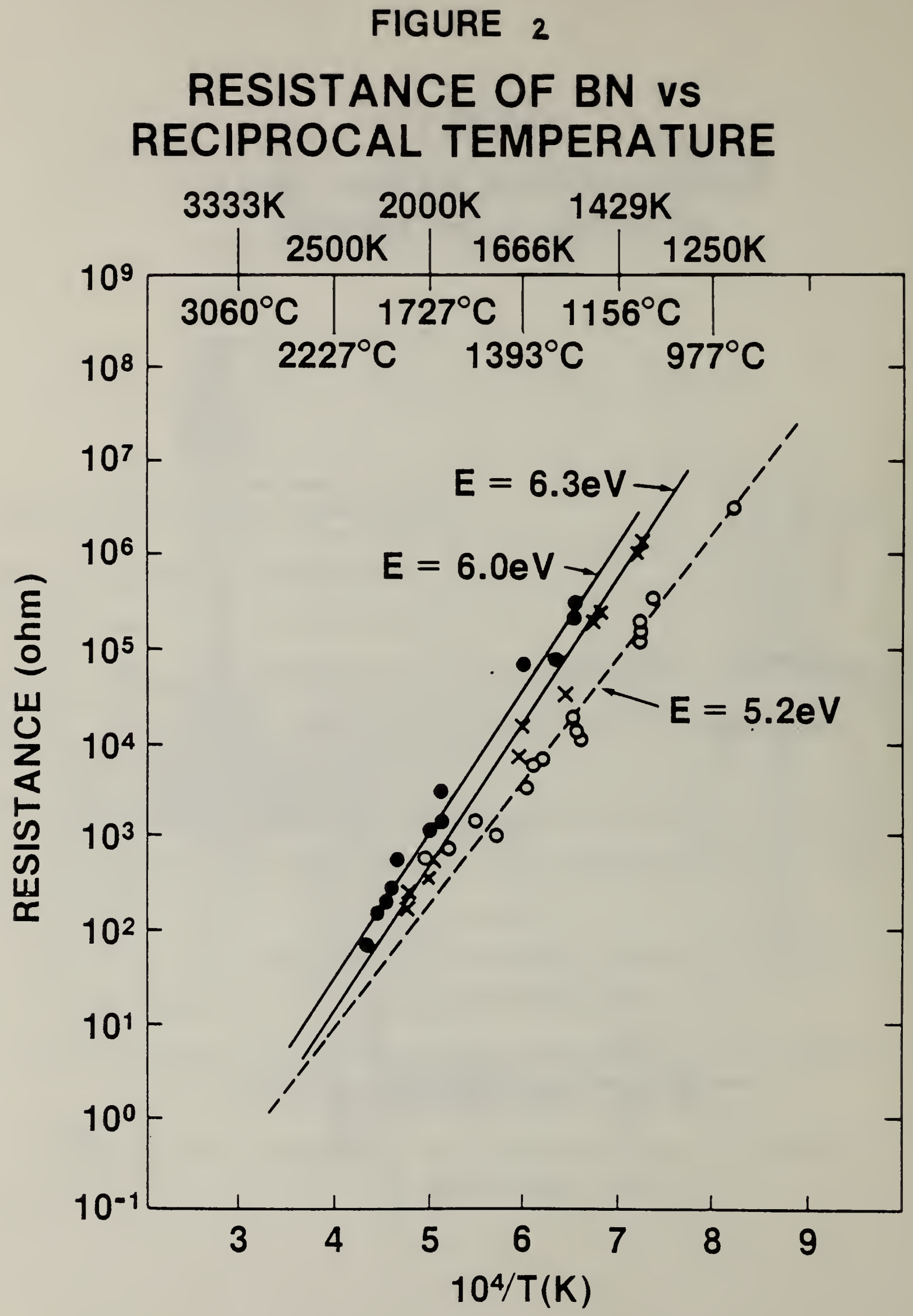




\section{FIGURE 3 \\ RADIOGRAPHS OF BN SAMPLE}

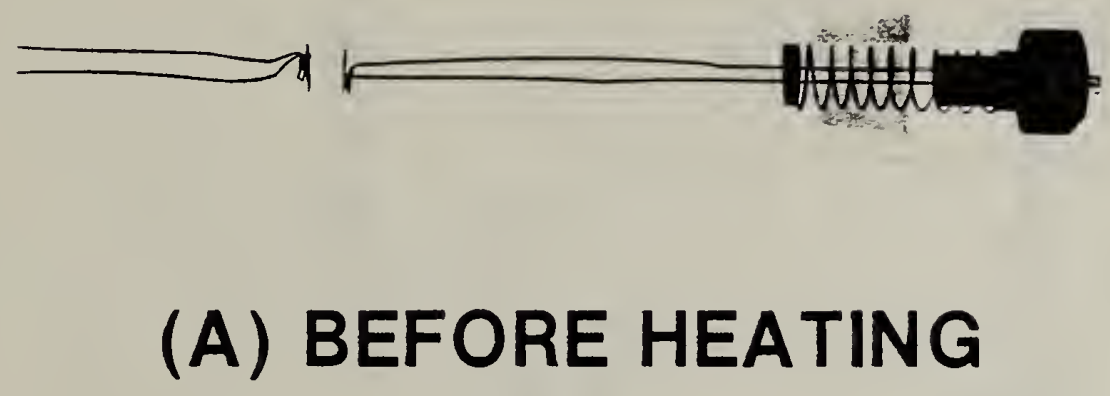

(A) BEFORE HEATING

$\therefore-\therefore=1 \frac{1}{20}$

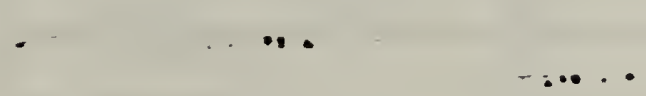

(B) AFTER HEATING 
FIGURE 4

PHASE DIAGRAM OF W-B SYSTEM

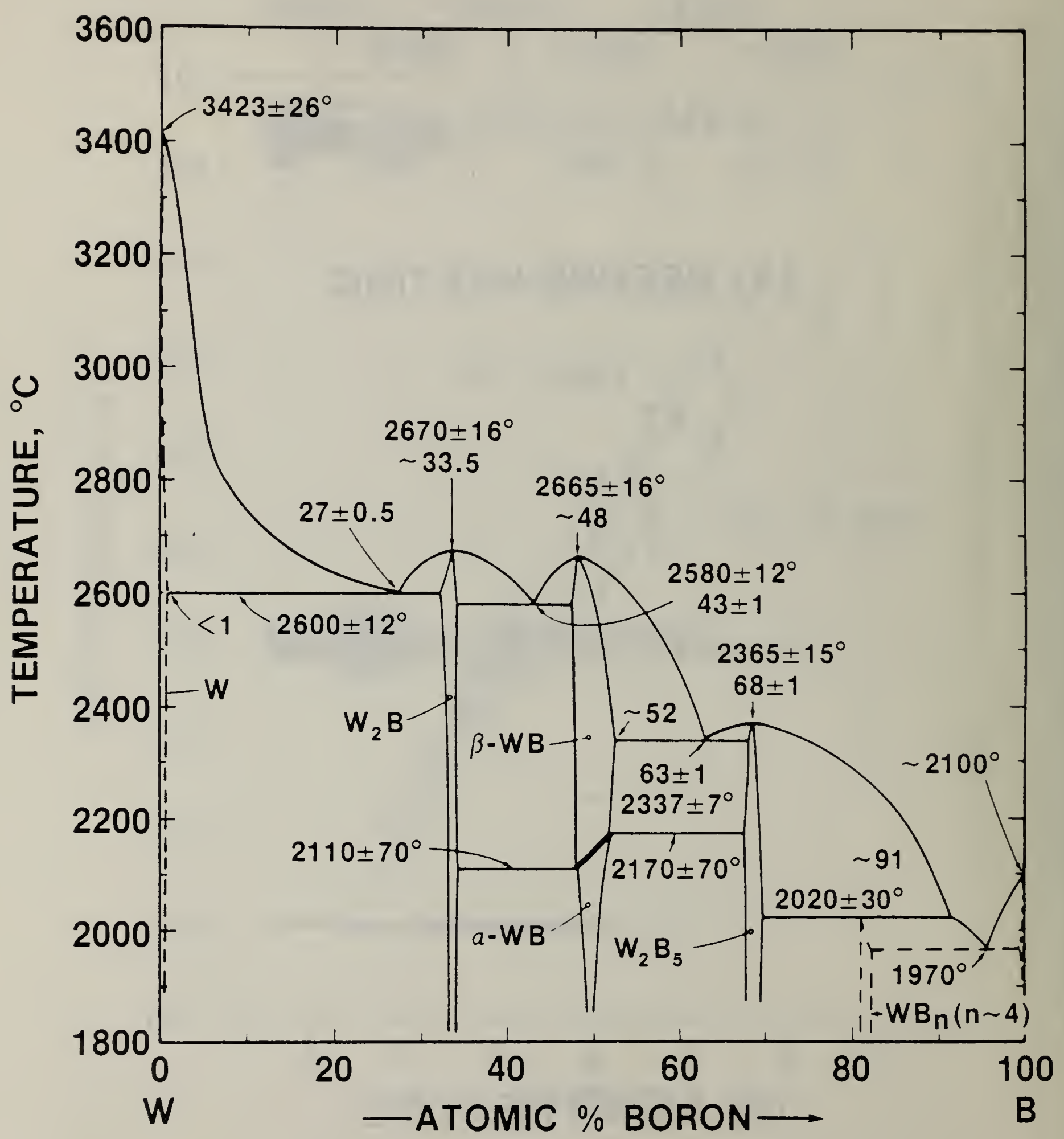




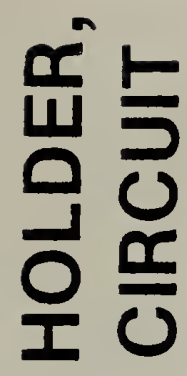

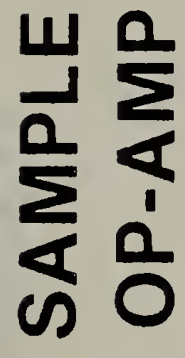

능 운

0

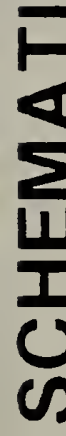

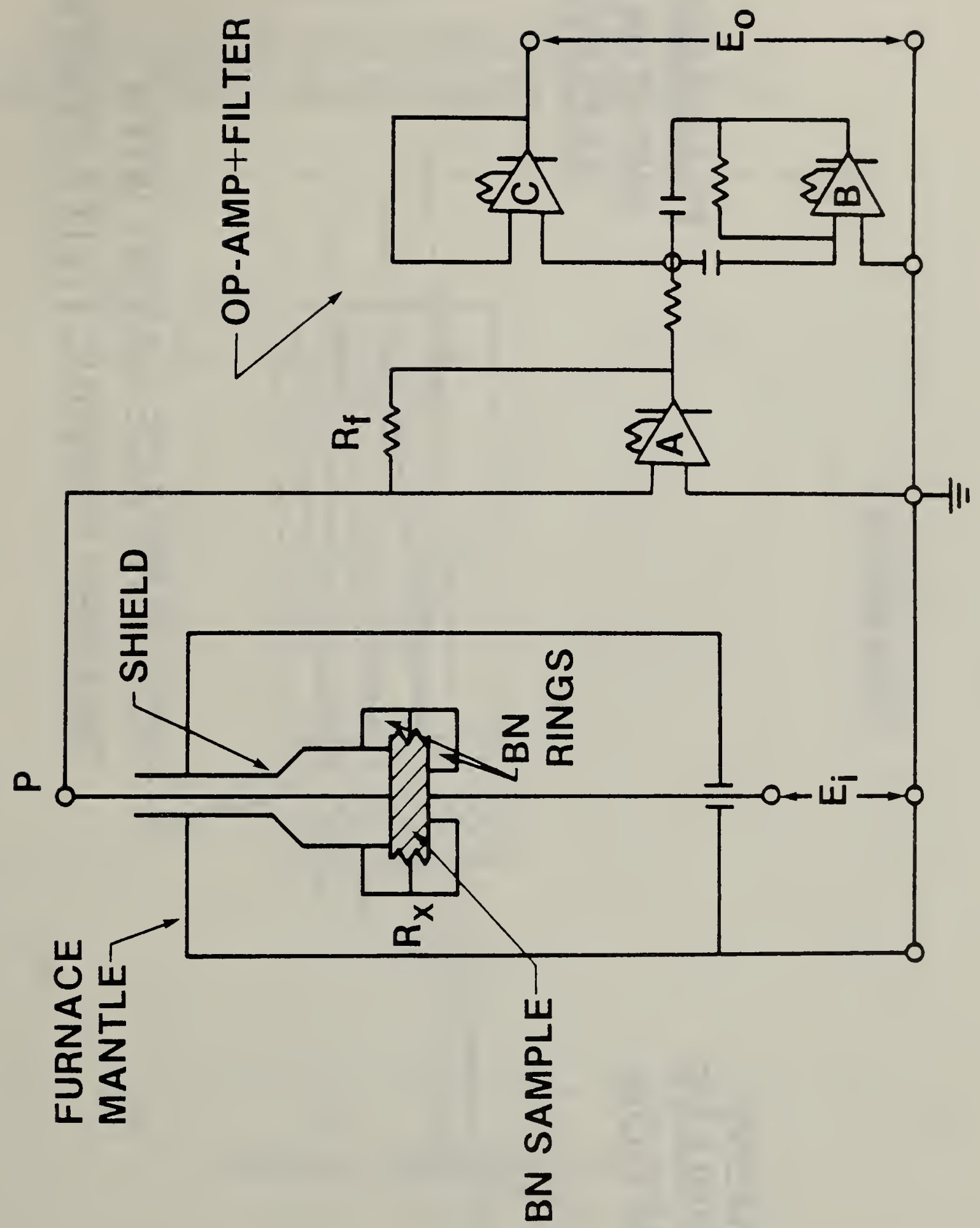




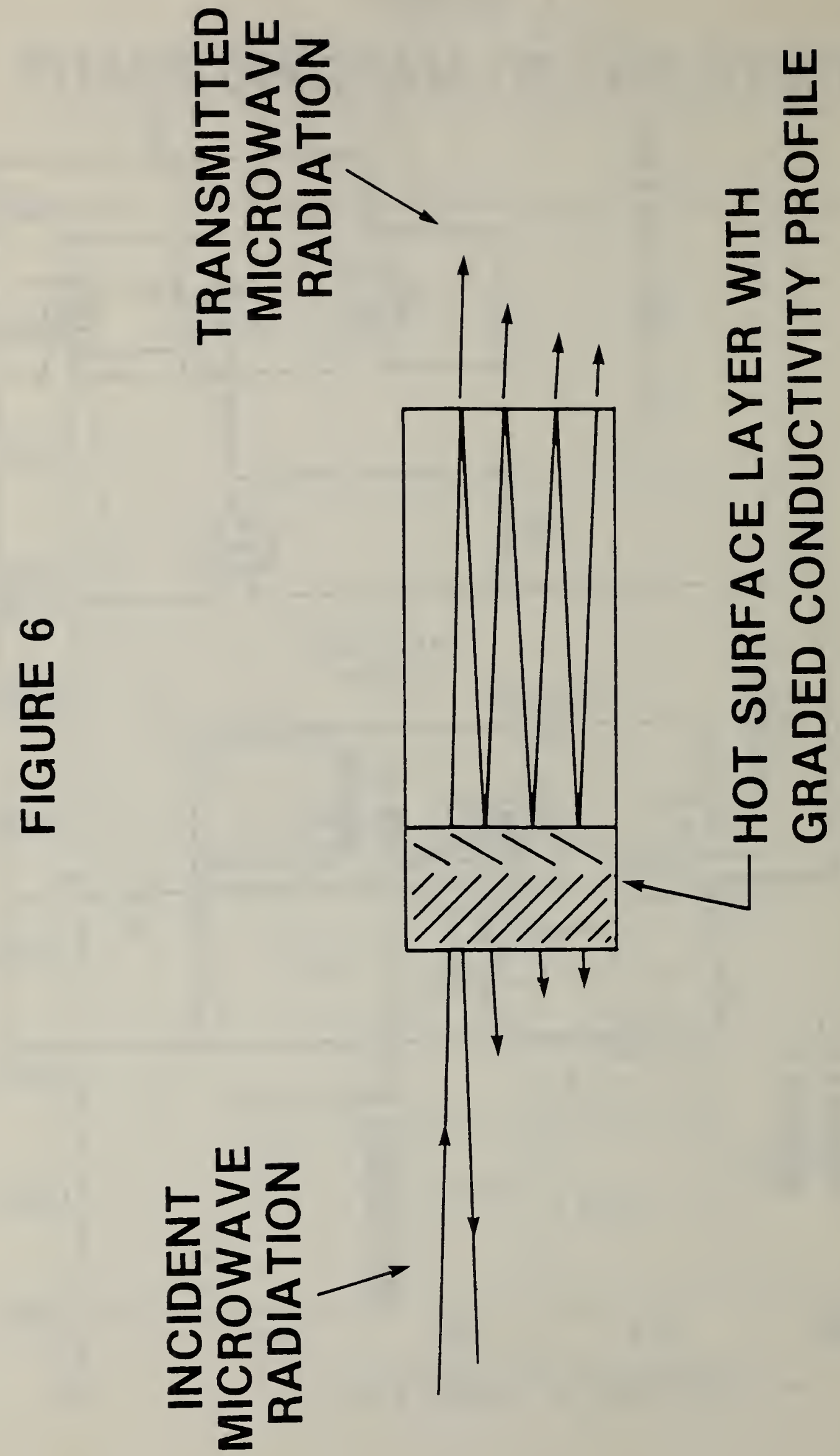


FIGURE 7

\section{CALCULATED TRANSMISSIVITY OF BORON NITRIDE MICROWAVE WINDOWS}

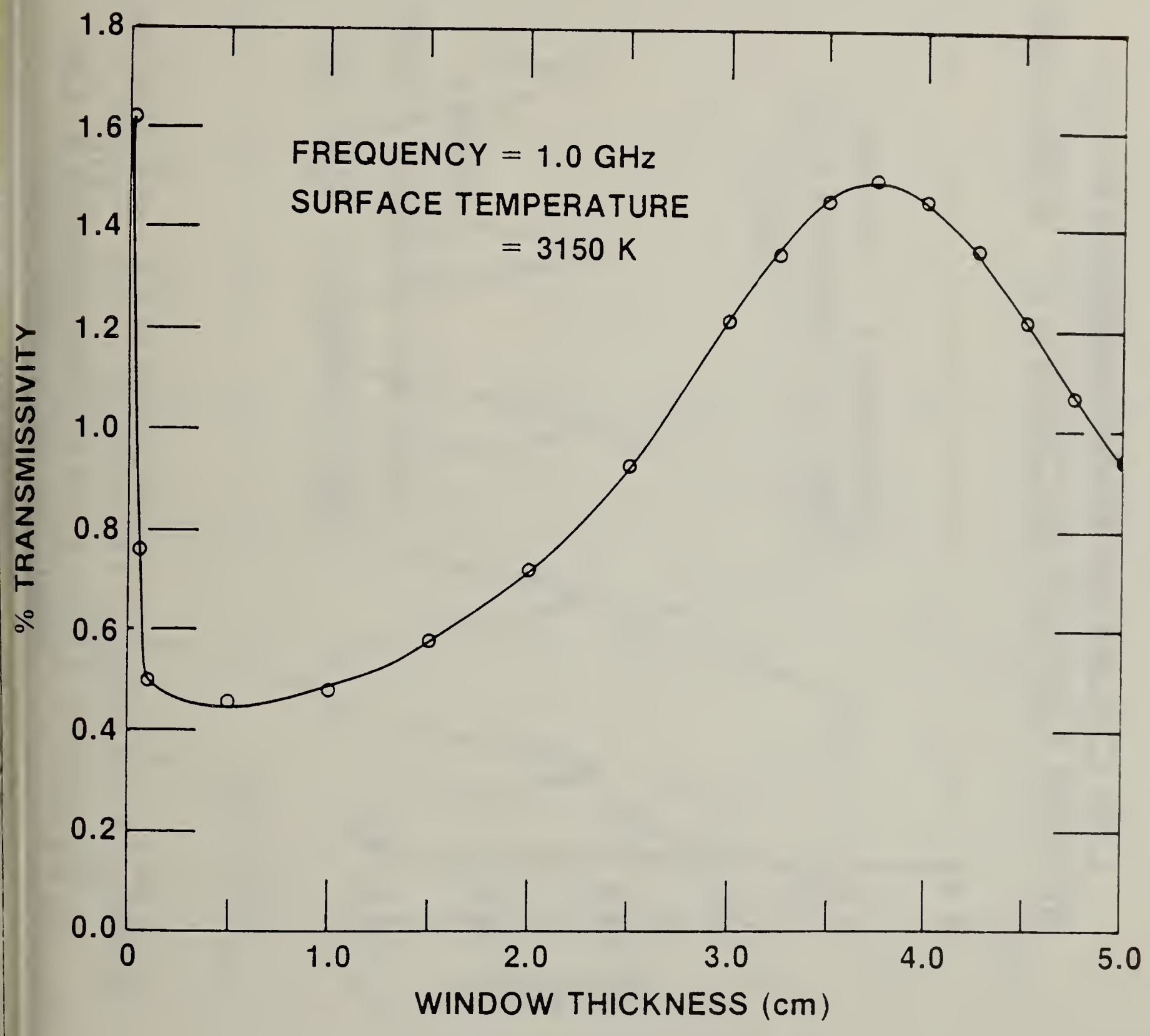




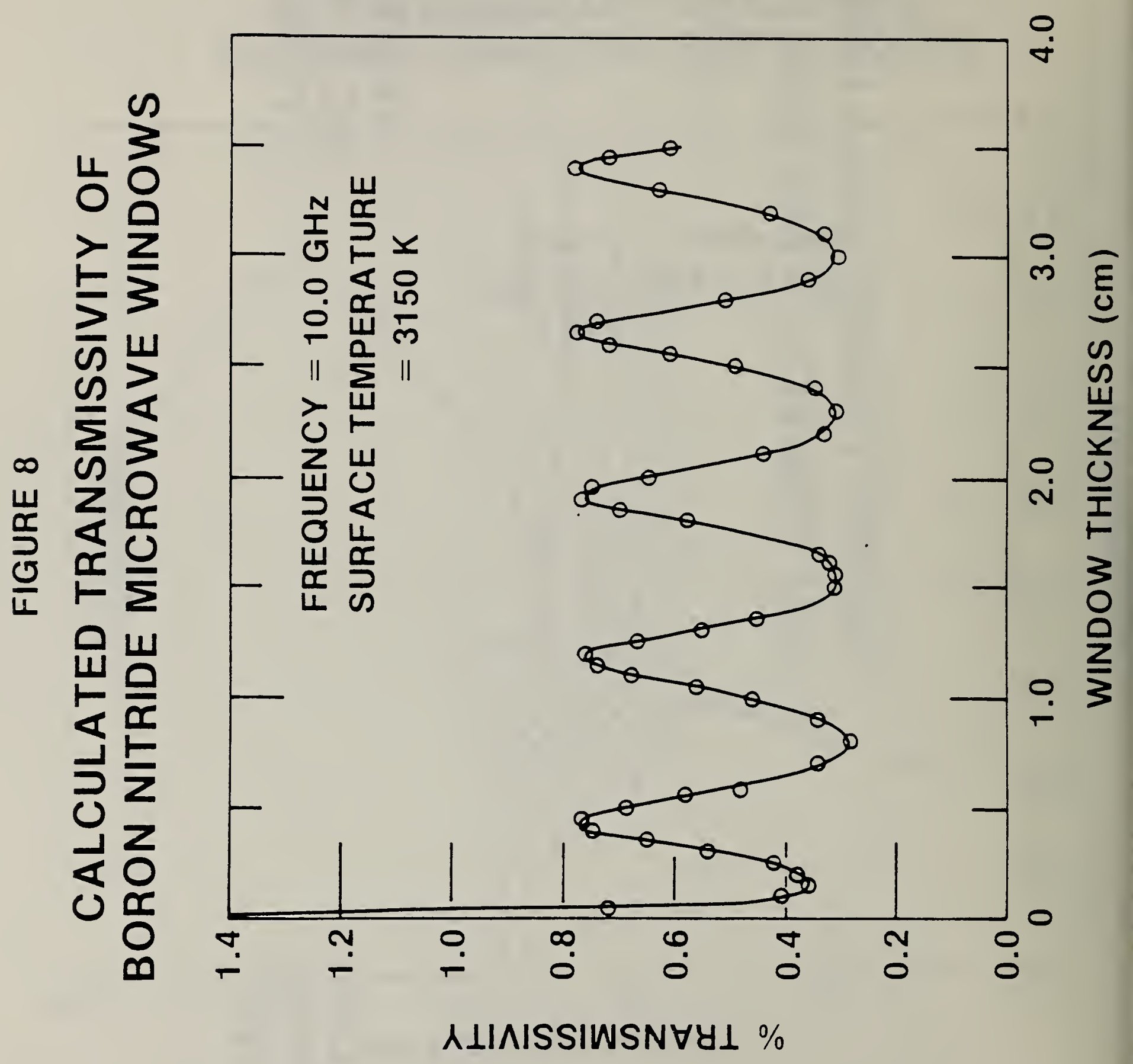




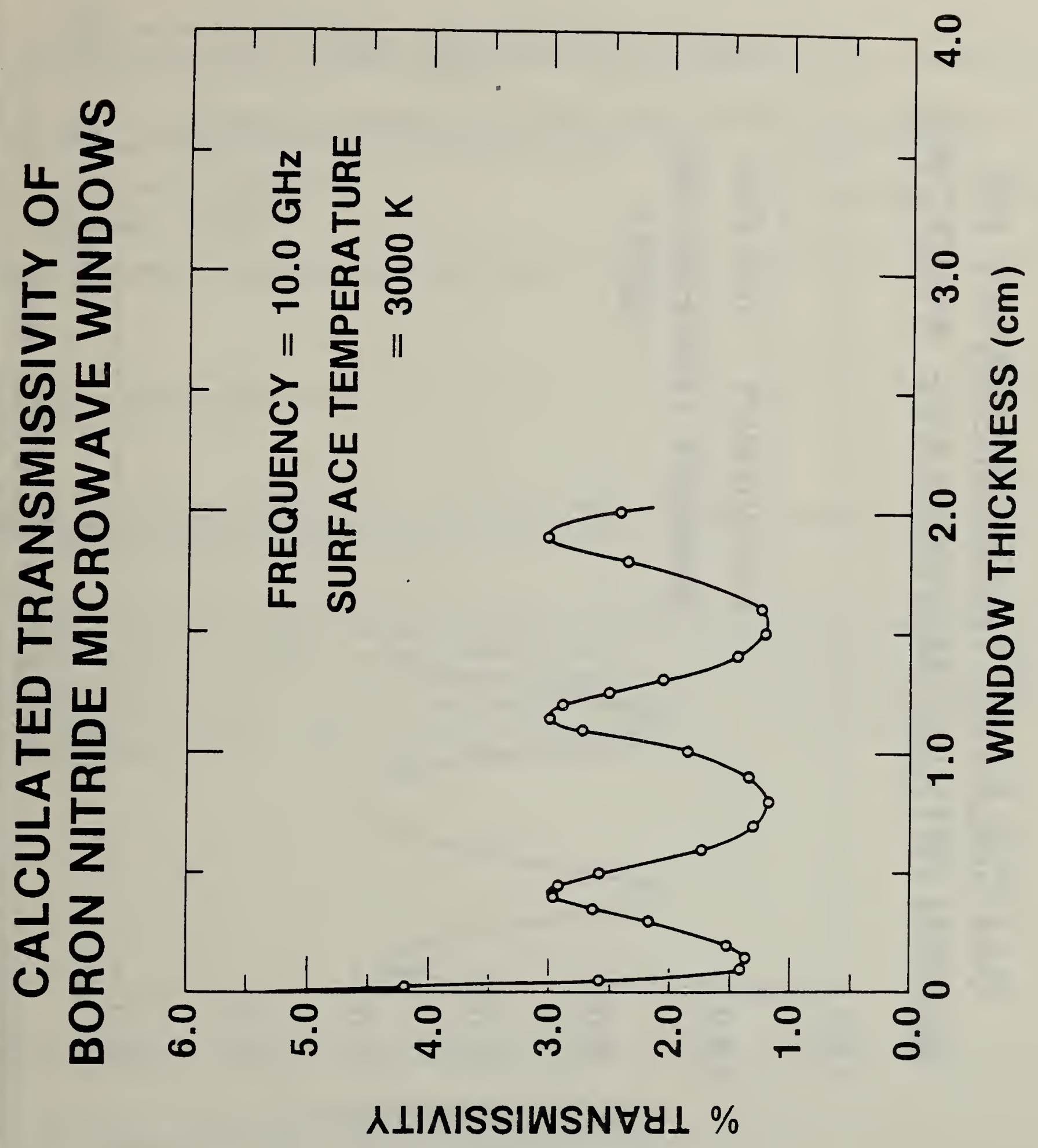




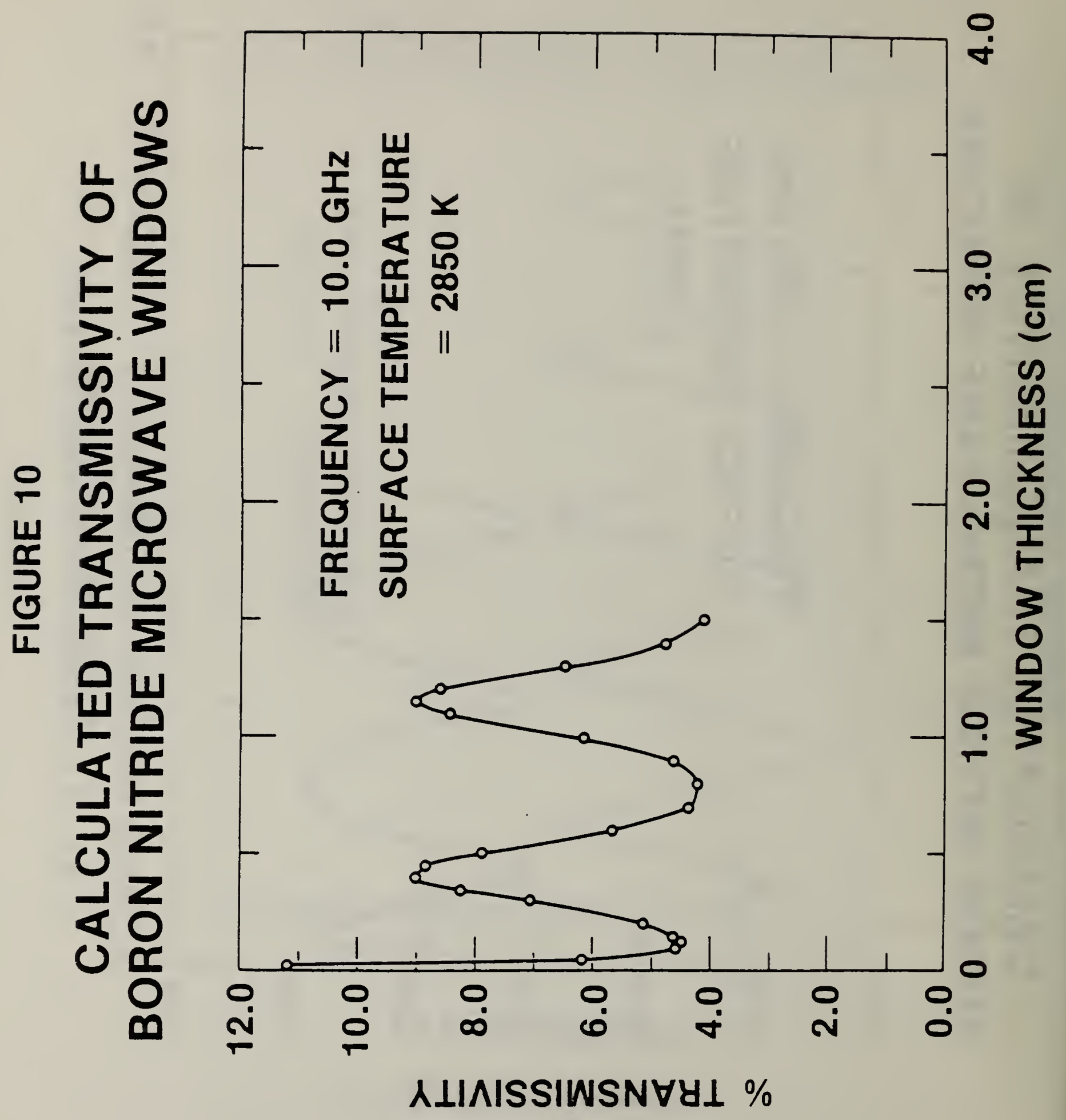


NBS-114A (REV. 2*8C)

U.S. DEPT. OF COMM.

BIBLIOGRAPHIC DATA

SHEET (See instructions)

1. PUBLICATION OR REPORT NO.

NBSIR $83-2646$

2. Performing Organ. Report No. 3. Publication Date

January 1983

4. TITLE AND SUBTITLE

"Characterization of ARV Antenna Window Material"

(Progress Report No. 2, October 1982; Defense Nuclear Agency)

5. $A U T H O R(S)$

H. P. R. Frederikse, A. L. Dragoo, A. H. Kahn, and W. R. Hosler

6. PERFORMING ORGANIZATION (If joint or other than NBS, see instructions)

7. Contracd Grant No.

NATIONAL BUREAU OF STANDARDS

DEPARTMENT OF COMMERCE

8. Type of Report \& Period Covereo

WASHINGTON, D.C. 20234

9. SPONSORING ORGANIZATION NAME AND COMFLETE MODRESS (Stret. City, STOLE, ZIP)

10. SUPPLEMENTARY NOTES

Document describes a computer program; SF-185, FIPS Software Summary, is attached.

11. ABSTRACT (A 200-word or less factual summary of most significant information. If document includes a significant bibliography or literature survey. mention it here)

Boron nitride is a major candidate for use as a microwave window material. To assess its feasibility for this purpose, it is essential to know the electrical and dielectric properties at high temperatures. This report discusses the experimental approach to electrical conductivity measurements above $1800{ }^{\circ} \mathrm{C}$ and presents some initial results. At the same time, computer calculations have been performed which have yielded values of the reflectivity, absorption, and transmission of boron nitride in the temperature range $2500-3000{ }^{\circ} \mathrm{C}$.

12. KEY WORDS (Six to twelve entries; alphabetical order; capitalize only proper names; ond separate key words by semicolons)

Boron nitride; Calculated transmissivity; Electrical conductivity; High temperatures; Microwave radiation

13. AVAILABILITY

Unlimited

- For Official Distribution. Do Not Release to NTIS

- Order From Superintendent of Documents, U.S. Government Printing Office, Washington, D.C. 20402.

14. NO. OF PRINTED PAGES

X_ Order From National Technical Information Service (NTIS). Springfield, VA. 22161

15. Price 


